\title{
Trois études stylistiques sur la clausule du trimètre iambique d'Aristophane (Vesp. 1219-1264 - Ran. 1365-1413 - Thesm. 846-928)
}

[Three Stylistic Studies on the clausula of Aristophanes' Iambic Trimeter: (Vesp. 1219-1264 - Ran. 1365-1413 - Thesm. 846-928)]

\author{
Marco Borea* \\ Université de Caen Normandie - \\ CRAHAM Centre Michel de Boüard
}

\begin{abstract}
Résumé : $\quad$ Dans le trimètre iambique, il y a des clausules tragiques et d'autres comiques. Ces deux types de clausules alternent dans les scènes para-tragiques d'Aristophane, dans le concours de chant des Guêpes, dans la pesée des vers des Grenouilles et dans la parodie de l'Hélène des Thesmophories.
\end{abstract}

\begin{abstract}
In the iambic trimeter, there are tragic line-ends and other comic ones. These two types of clausulae alternate in para-tragic scenes of Aristophanes, in the song competition of the Wasps, in the line weighing of the Frogs and in the Helen parody of the Thesmophoriazusae.
\end{abstract}

Mots-clés : $\quad$ trimètre iambique, para-tragédie, Aristophane, clausule

Keywords: $\quad$ iambic trimeter, para-tragedy, Aristophanes, line-end

Recepción: $\quad 27 / 03 / 2020$

Aceptación: $\quad 25 / 06 / 2020$

Cette étude de métrique stylistique prend en considération trois scènes en trimètres iambiques tirées des comédies d'Aristophane, pour y déceler les effets stylistiques de la clausule. Il s'agira, dans un premier temps, d'aborder les trimètres insérés parmi les mètres lyriques du chœur dans le concours de chant cocasse qui oppose Philocléon et Bdélycléon dans les Guêpes (Vesp. 1219-1264). Dans un deuxième temps, on se concentrera sur les trimètres du débat, en l'espèce ceux de la pesée des vers à la fin de l'agôn poétique entre Eschyle et Euripide dans les Grenouilles (Ran. 1365-1413), et ceux de la section para-tragique qui encadre les citations que Mnésiloque et Euripide débitent devant l'assemblée des femmes des Thesmophories (Thesm. 846-928) ${ }^{1}$.

"Dirección para correspondencia: Centre Michel de Boüard, Craham - Université de Caen Normandie. Esplanade de la Paix. 1400 Caen (Francia). E-mail: marco.borea@hotmail.it

${ }^{1}$ Pour le texte et les traductions d'Aristophane, nous avons suivi l'édition de V. Coulon des Belles Lettres. 


\section{LE CONCOURS DE CHANT ( VESP. 1219-1264)}

L'entrée de Philocléon dans la société se résout en un véritable désastre. Après s'être fait prier pour s'habiller et sortir (v. 1122-1173), le vieux bourru est taquiné sur son langage et sa capacité à tenir un discours sérieux (v. 1174-1218).

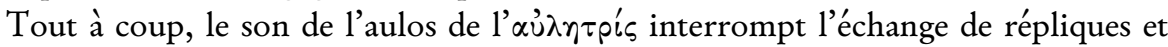
marque le début d'une nouvelle section (v. 1219-1264), au cours de laquelle Bdélycléon et Philocléon se lancent dans une sorte de concours de chant. Père et fils prennent à tour de rôle la parole pour entonner des motifs populaires, très en vogue lors des banquets. Tous ces chants se déroulent selon des mètres différents. Dans le premier chant, une sorte de chant hérö̈que en l'honneur des tyrannicides Harmodios et Aristogiton, Bdélycléon donne le la et est suivi par la voix du père qui reprend les paroles tout en les tournant en ridicule. Il est assez curieux de remarquer que le vieux père détourne uniquement les mots et le sens de la chanson, mais non pas le mètre qui reste un hendécasyllabe phalécien ${ }^{2}$. Peu de vers après, c'est à Philocléon d'entonner un autre chant, une parodie d'un fragment d'Alcée (fr. $141 \mathrm{~L}-\mathrm{P})$ à l'adresse de Cléon ${ }^{3}$. À quoi Bdélycléon réplique avec un asclépiade

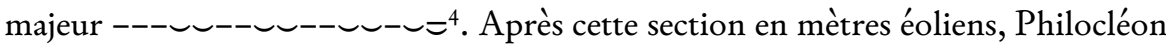

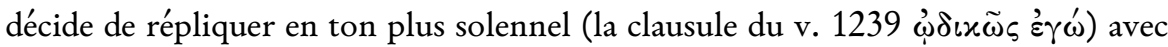
deux côla qu'on retrouve ici et là dans la tragédie ${ }^{5}$. Les deux derniers motifs tournent en ridicule Eschine, un fanfaron d'une grande renommée ; ils sont chantés, comme

${ }^{2}$ L'hendecasyllabe phalécien est un mètre éolien qui prend le nom du poète alexandrin Phalécos (III siècle avant notre ère) et présente le schéma suivant : $x \times-\smile \smile-\smile-\smile--$. Vesp.

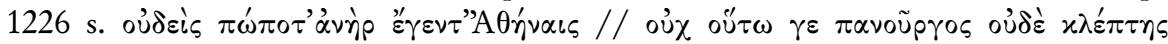
(Bdélycléon) Jamais homme ne fut, dans Athènes... // (Philocléon) ...à ce point malfaisant et voleur!

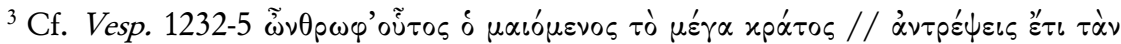

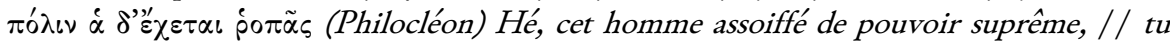
bouleverseras un jour la cité, son sort est en balance. Philocléon convertit presque tous les mots éoliens dans leur forme attique, à l'exception de $\tau \dot{\alpha} \nu$, l'article $\dot{\alpha}$ et le substantif $\hat{\rho}_{0} \pi \tilde{\alpha}_{\varsigma}$; le mètre est une sorte d'expansion du glyconien de quinze syllabes.

${ }^{4}$ L'asclépiade majeur est un mètre éolien qui dérive de l'asclépiade mineur $\times x-\smile \smile-\smile-$ $\smile-=$ avec l'insertion d'un choriambe ; cf. M.C. Martinelli, 1995, pp. 233-245; M. Steinrück, 2007, p. 64 s. En latin, pour l'emploi de ce vers dans des asinarthètes ou $x \alpha \tau \dot{\alpha}$

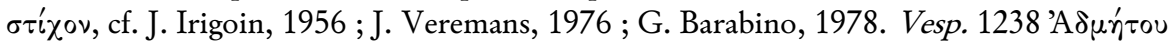

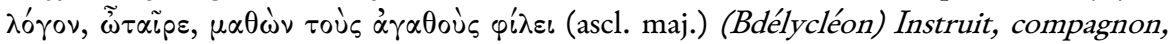
de l'histoire d'Admète, il aime les bons.

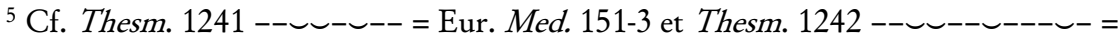
Eur. Med. 158 ainsi que B. Zimmermann, 1985, pp. 51-53 et 1987, p. 32. 
au début (v. 1226 s.), à tour de rôle par le fils et le père ${ }^{6}$. Ce qu'il est curieux de remarquer ici est qu'au changement de ton et de contenu des deux répliques, notamment la version sérieuse du souhait entonné par Bdélycléon et la réplique déplacée et grossière de Philocléon, correspond aussi le changement de mètre, les dochmies propres à la tragédie et le moins lyrique hendécasyllabe phalécien.

Tous ces passages sont entremêlés par des trimètres qui étaient probablement récités et, par conséquent, opposés aux côla lyriques des chansons. À présent, si l'on s'intéresse à la clausule de ces trimètres, on voit très aisément que, quant à sa structure, elle se conforme assez rapidement à ce mélange de rythmes différents. Par exemple, trois fois ${ }^{7}$ sur cinq, le trimètre qui précède le motif chanté se termine sur un hiatus interlinéaire saisissant qui signale le changement du récitatif au lyrique et qui semble spécialement destiné à marquer les arrêts du rythme. Dans tous les cas, il est facile de voir où le trimètre finit et où le chant débute $(B=$ Bdélycléon ; $\mathrm{P}=$ Philocléon) :

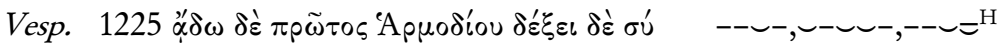

(B) Je chante le premier de l'" Harmodios », tu reprendras toi!

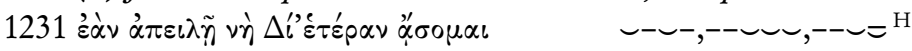

(P) S'il me menace, par Zeus, j'en chanterai une autre!

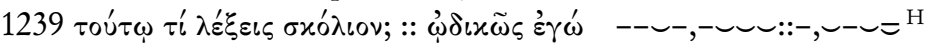

(B) À celui-là par quelle chanson répliqueras-tu ? :: (P) Lyrique moi !

L'hiatus intervient deux fois après le pronom personnel emphatique ( $v$. $1225,1239)$ et une fois lors de la première personne du futur $\alpha$ ć $\sigma o \mu \alpha \iota$ qui indique aussi la prise de position (maladroite) de Philocléon. En outre, les clausules des trimètres récités tissent un réseau complexe d'échos verbaux entre un chant et l'autre. À titre d'exemple, on peut prendre le cas de la clausule homophone $\mathrm{K} \lambda \boldsymbol{\varepsilon}^{\prime} \omega \nu$ / $x \alpha \lambda \tilde{\omega} \varsigma:$

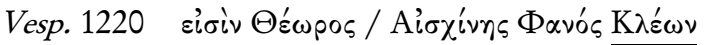

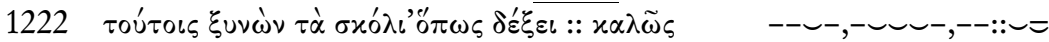

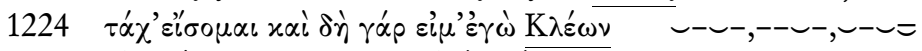

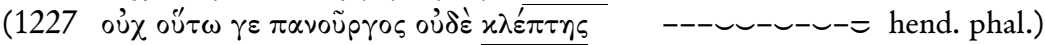

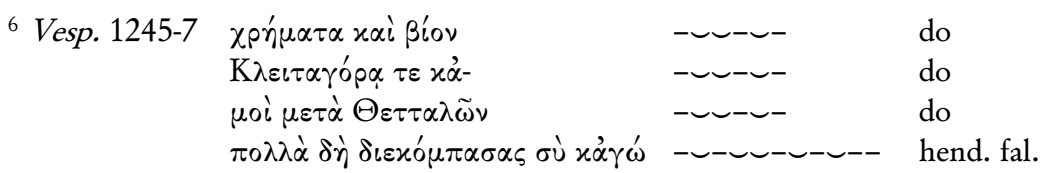

${ }^{7}$ Cf. Thesm. 1225,1231 et 1239. 
Trois études stylistiques sur la clausule du trimètre iambique d'Aristophane...

En même temps, la clausule du premier chant de Philocléon permet de reconnaitre toujours des finales qui riment avec les trimètres précédents (v. 1227). Les correspondances des clausules à distance sont, à un tel endroit de la comédie, d'autant plus frappantes que l'un des invités du banquet est justement Cléon, la cible principale de la pièce et que les dissyllabes finaux, clausule plus tragique que comique, demeurent absolument minoritaires dans toute la scène (huit seulement au total vis-à-vis de dix trisyllabes). Au v. 1220, le nom de Cléon figure au terme de l'énumération des convives qui font partie de son entourage; il est repris dans les paroles de Bdélycléon qui débute le concours de chant en faisant semblant d'être Cléon lui-même. L'écho le plus frappant semble résider dans la clausule du v. 1227, où Philocléon rabaisse le ton solennel du fils, tout en détournant les paroles. Ainsi, l'épithète assignée à Harmodios peut aussi (et volontairement) être rapportée à Cléon, en vertu de l'homophonie de l'adjectif $\chi \lambda \varepsilon^{\prime} \pi \tau \eta \varsigma$ avec le nom K $\lambda \varepsilon^{\prime} \omega \nu$. Mais les consonances ne s'arrêtent pas à cet écho entre dissyllabes et s'étendent aux autres

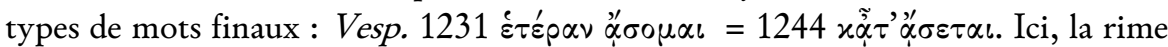
clausulaire concerne un mot-clé de ce véritable « karaoké » convivial, notamment le verbe $\alpha \alpha \delta \omega$; la fin du v. 1244 rime aussi avec la clausule du vers précédent (v. $\left.1243 \delta^{\prime} \xi_{\varepsilon}^{\prime} \varepsilon \alpha_{l}\right)$. Un autre écho, plus subtil, intervient dans les deux trimètres qui

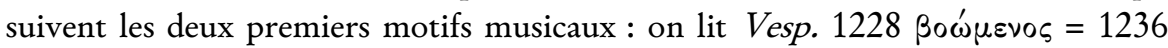
$\varkappa \alpha \tau \alpha \varkappa \varepsilon i ́ \mu \varepsilon v o \varsigma$, les deux vers se terminant sur une forme participiale. Encore, les

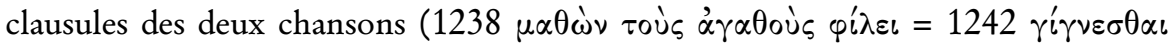
$\varphi(\lambda \circ v)$ finissent sur le sujet de l'amitié et la fraternité, en alternant version sérieuse (celle de Bdélycléon du v. 1238) et version burlesque (celle de Philocléon du v. 1242).

Le passage qui suit le concours de chant au banquet mérite aussi une analyse spéciale ; en effet, c'est précisément après la réplique aussi grossière que véridique de Philocléon que Bdélycléon propose alors de changer d'ambiance et d'aller dîner chez Philoctémon, un autre débauché qui tenait table ouverte de toutes parts (v. 1250

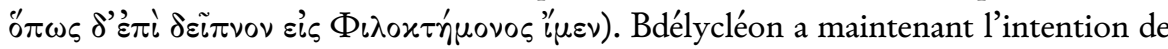
faire boire son père au cours du dîner pour qu'il puisse être guéri de sa «tribunalite ». À quoi, dans un premier moment, Philocléon répond énergiquement :

Vesp. $1252-5$

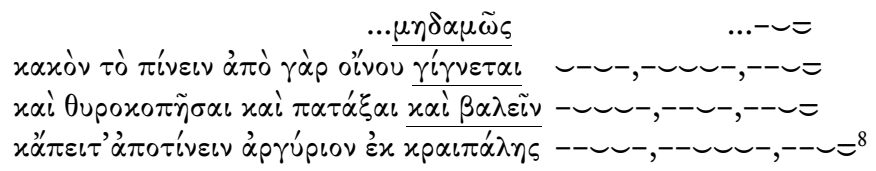

${ }^{8}$ Nullement, // ce n'est pas bien de boire : c'est du vin qui résultent // les portes cassées, les coups donnés, les pierres lancées, // et, ensuite, l'argent à payer, une fois le vin cuvé. 
Le refus de l'alcool manque de crédibilité et fait au contraire ressortir à nouveau le caractère bouffon et ridicule de Philocléon. En effet, sa plaidoirie en faveur du renoncement à l'alcool se résout en une longue énumération des risques et des inconvénients auxquels sa consommation non contrôlée peut facilement emmener. Les clausules trisyllabiques réitérées sans cesse soulignent toute la grossièreté et la gaucherie du personnage ; le pont de Porson est violé pour la troisième fois consécutive (v. 1252-1254). Pour répliquer à autant de maladresse, son fils Bdélycléon échafaude un discours qui vise à justifier la consommation

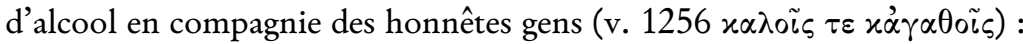
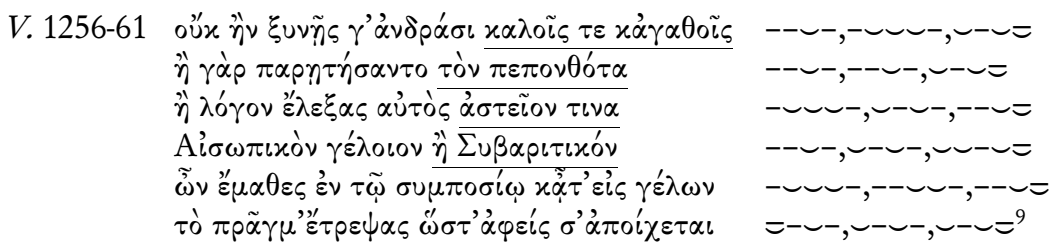

Les clausules de ces passages font se succéder une série de mots longs de sept et cinq syllabes qui confèrent au parler de Bdélycléon plus de crédibilité et de force persuasive, d'autant plus qu'une telle concentration de mots longs contigus est tout à fait inhabituelle dans la pièce. Ce n'est qu'aux deux derniers vers que la longueur des mots finaux se réduit et que les clausules admettent un trisyllabe et un tétrasyllabe. Il ne passera pas inaperçu que dans ce passage, où le ton devient plus sérieux, le pont de Porson n'est jamais violé sauf au v. 1260, avec toutefois l'élision. L'autorité du langage du fils finit rapidement par convaincre l'immature Philocléon et pour le traîner

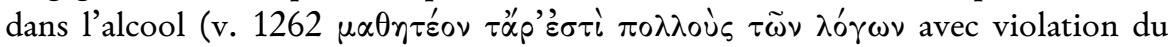
pont de Porson) ; les deux, sûrs de leurs actions, s'engagent alors sur le chemin qui mène

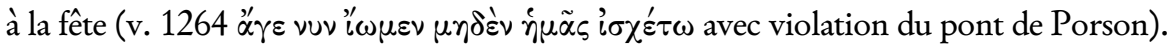
Par conséquent, ce fameux dîner sera alcoolisé au point que Philocléon reviendra complétement éméché. On voit donc à quel point le jeu de miroir entre violation et respect du pont de Porson reflète l'alternance de registre tragique et comique.

Les trimètres insérés dans le concours lyrique Bdélycléon / Philocléon ne finissent jamais de surprendre à cause de l'extraordinaire réseau d'échos qu'ils entretiennent avec les côla lyriques des chansons entonnées, en exploitant toutes les ressources de

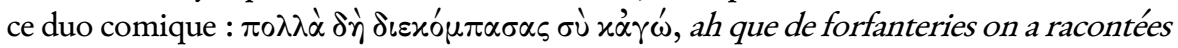
...toi et moi!

${ }^{9}$ Non, si tu es avec des gens comme il faut, // car ou ils t'excusent auprès de l'offensé, // ou toi-même tu racontes quelque histoire spirituelle, // une blague à la manière d'Ésope ou de Sybari, // parmi celles que tu as apprises à table; puis, tu tournes // la chose en plaisanterie, si bien qu'il te libère et s'en va. 
2. LA PESÉE DES VERS ( $R A N$. 1365-1413)

Dans les Grenouilles, l'arrivée sur scène de la balance censée déterminer le vainqueur de l'agôn va changer fortement la nature du débat. En réalité, la pesée des vers ne débouchera sur aucun jugement effectif et il faudra attendre le débat final, à contenu politique, pour voir attribuer la victoire à Eschyle. On abandonne alors l'examen technique et méthodique des prologues et des types de mélodies pour entrer dans une scène surréaliste où les couplets de trimètres, cités par les deux Tragiques, sont pesés sur la balance l'un après l'autre. Par une inversion du motif homérique de la pesée du sort léger d'Achille opposé à la lourdeur du sort d'Hector, dans les Grenouilles gagne le vers qui pèse le plus. Le mot léger ne fait qu'évoquer des réalités invisibles ou petites tandis que le mot lourd matérialise une réalité évanescente.

Dionysos et Eschyle sont d'accord sur le fait qu'il faut interrompre le concours des mélodies et passer à autre chose (v. $1364 \pi \alpha u ́ \sigma \alpha \sigma \theta \circ \nu$ 光 $\delta \eta \tilde{\omega} \nu \mu \varepsilon \lambda \tilde{\omega} \nu$

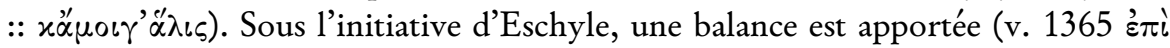

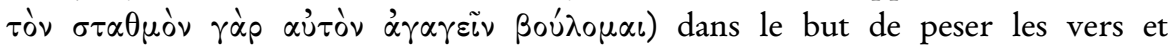
d'affronter publiquement son pire adversaire poétique. Le changement de ton de la joute poétique est marqué par l'interruption brusque de l'expression juridique $\varphi \omega \rho \alpha ́ \sigma \omega$, qui suit l'invocation à Hécate (v. 1362 s.) du chant multiforme parodié par Eschyle. Au gré de leurs envies, les deux adversaires déclarent à l'unisson (v.

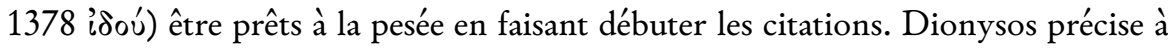

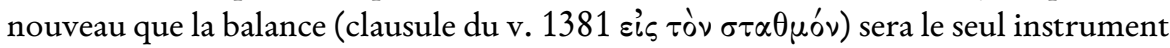
de jugement. Les couplets de trimètres viennent donc scander les étapes de cette pesée :

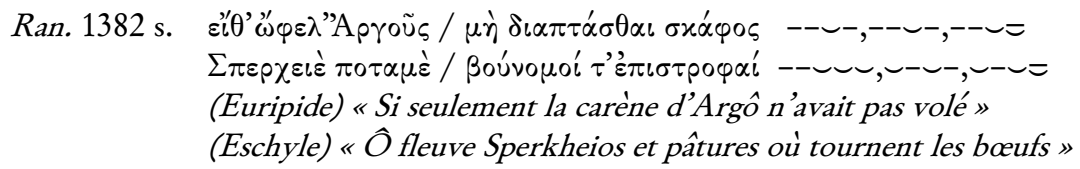

Pour les deux premiers trimètres, Euripide choisit le prologue de sa Médée; il s'agit de la première protase d'une longue phrase hypothétique introduite par la nourrice qui se plaint de la venue du navire Argô et de Jason en Colchide, patrie de Médée. Eschyle, quant à lui, cite un trimètre de son Philoctète (fr. 249 Radt), où le poète invoque le fleuve et les pâturages de la Thessalie, patrie de Philoctète. Une fois les deux trimètres posés sur la balance, Dionysos décrète la victoire d'Eschyle :

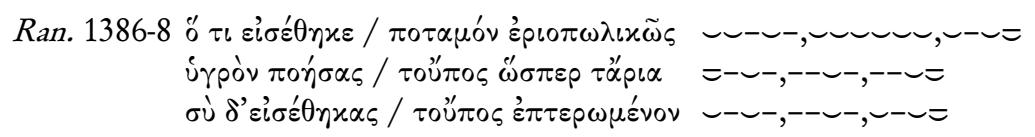


Parce qu'en bon marchand de laine, il y a mis un fleuve, et composé un vers mouillé, comme on fait avec la laine, toi, le vers que tu as mis avait des ailes

Seul le nom du fleuve a fait pencher la balance et le dieu ne tient pas compte

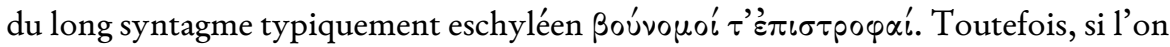
examine les deux clausules dans ce face-à-face poétique, on constate une disproportion dans la brièveté de la clausule dissyllabique oxáyos de l'incipit de la Médée vis-à-vis de la longueur du syntagme grandiloquent pâtures où tournent les boufs. La discrimination se fait alors non seulement en termes du contenu, comme Dionysos le prétend, mais tout autant sur la base de la clausule, véritable point sensible du rythme du vers. Le deuxième couplet voit s'opposer deux trimètres tirés respectivement de l'Antigone et de la Niobé:

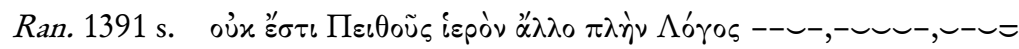

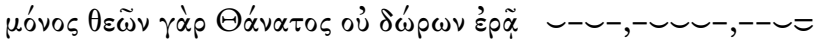
(Euripide) "Il n'est d'autre temple de la Persuasion que le Discours » (Eschyle) "Seul parmi les dieux, le génie de la mort n’aime pas les dons»

La comparaison de ces deux vers s'appuie sur une distribution parallèle des mots. En effet, tous les deuxièmes hémistiches débutent sur une résolution du sixième élément; deux noms propres désignent deux prosopopées, $\Lambda$ ó $\Theta_{\alpha}^{\prime} \nu \alpha \tau o \varsigma$, antithétiques non seulement quant à leur localisation mais aussi quant aux concepts opposés qu'ils évoquent. En outre, pour ce qui est des clausules, Euripide finit par être à nouveau surclassé par son adversaire. Si Dionysos justifie la nouvelle défaite d'Euripide en alléguant la lourdeur incontestable de la mort, il est toutefois probable que l'échec d'Euripide soit causé par une mauvaise facture des vers. Le trimètre qu'il prononce se termine en effet sur un trisyllabe (mot métrique), précédé par une voyelle fort probablement brève. Pour sa part, Eschyle répond avec une clausule impeccable et une construction aussi simple que linéaire composée de substantif + verbe. Ce deuxième échec ne passe pas inaperçu et donne l'occasion à Dionysos pour couvrir de critiques le perdant, Euripide, et pour l'inciter ainsi à répliquer (v. 1396-8). Les vers soumis à troisième pesée affichent la même finale :

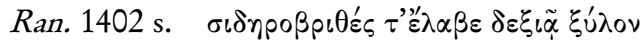

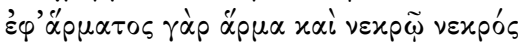

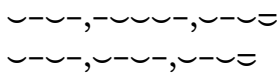

(Euripide) "Et de sa main droite, il saisit un bois lourd de fer " (Eschyle) "Car char sur char et cadavre sur cadavre »

Cette fois-ci, Euripide récite un trimètre du Méléagre (fr. $18 \mathrm{~J}-\mathrm{VL}$ ) débutant sur un long composé en style eschyléen et se terminant sur un schéma 
composé d'un mot de deux demi-pieds + trois demi-pieds + deux demi-pieds, qui est complété, à son tour, par un trimètre du Glaucos de Potnies (fr. 38,1 Radt). Les deux trimètres pesés sont en correspondance sémantique et structurale étroites, puisque tous les deux se concluent sur un trisyllabe suivi d'un dissyllabe. En dépit de la ressemblance extérieure, c'est encore à Eschyle de l'emporter sur son adversaire en façonnant un trimètre plus lourd et construit autour d'un polyptote réussi. L'amélioration d'Euripide dans sa technique poétique n'échappe pas au juge

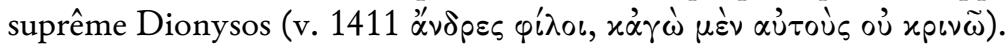

$\mathrm{Si}$, aux dires de Dionysos, cette pesée des vers ne semble concerner que le vocabulaire employé, il y a tout lieu de croire qu'elle trouve aussi son point d'appui sur la clausule, qui est aussi le repère rythmique culminant du vers.

\section{ENTRE RÉALITÉ ET FICTION ( THESM. 846-928)}

En écopant de la peine infligée par les femmes, le parent Mnésiloque a l'idée d'attirer son compagnon Euripide, complètement dépité et désemparé, en parodiant l'un de ses drames. La sélection d'un titre s'impose dès le début (Thesm. 846 s.). Dans un premier temps, le choix tourne autour du Palamède, défini froid ¿uxpóv, même si, subitement, Mnésiloque se rétracte et propose l'Hélène, étiquetée

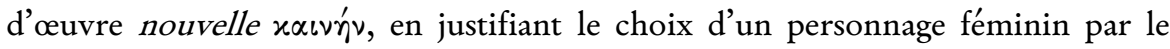
déguisément qu'il porte. Dès lors, commence une scène parodique fort plaisante au cours de laquelle trois voix alternent : celle du parent contrefaisant Hélène et celle d'Euripide imitant son époux Ménélas ; en contre-chant des citations lyriques, la troisième femme de l'assemblée intervient plusieurs fois pour couper la parole et ramener les deux prisonniers à la réalité. Le dialogue entre les trois pivote autour de ce contraste entre le lyrisme tragique, filtré, d'une part, à travers les yeux de la fiction cocasse et burlesque, d'Euripide / Ménélas et Mnésiloque / Hélène et, de l'autre, la brutalité de la réalité dans laquelle la femme veut les plonger.

Ainsi, dans une scène qui mêle citations tragiques aux caquetages de la femme, la clausule semble retracer à point nommé toutes les oscillations stylistiques d'un registre solennel à un parler grossier et déplacé. C'est donc précisément à la clôture des trimètres de cette première scène de parodie que l'on aborde l'enquête métrique $^{10}$.

${ }^{10}$ Sur les deux colonnes suivantes, on a répertorié, à gauche, les vers faisant partie d'une parodie, littérale ou remaniée, de l'Hélène, où Euripide et Mnésiloque prennent une voix de fausset, à droite, les trimètres dont les clausules sont attribuées à la femme ou aux répliques du duo comique sortant de leur rôle d'acteur et revenant au ton naturel de leur voix. Avec un astérisque, on a noté les vers des citations coupées par le passage brusque au registre comique : 
La clausule suit, pas à pas, ou mieux, trimètre après trimètre, le décalage continu entre fiction et réalité, dans un dérapage progressif, et s'adapte petit à petit au langage grivois de la femme. Au v. 855, Mnésiloque entonne le prologue de la tragédie, en contrefaisant en fausset la voix d'Hélène et en arborant le ton solennel propre à un incipit tragique. Le passage mérite d'être cité intégralement :

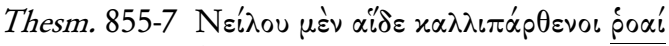

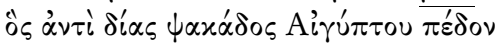

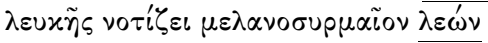

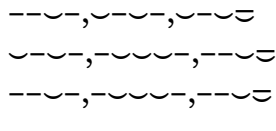
"Du Nil voici les courants virginaux, du fleuve qui, remplaçant l'eau du ciel, mouille le sol d'Égypte la blanche pour le peuple à la noire syrma»

La citation est presque littérale ${ }^{11}$, sauf le dernier trimètre, pour lequel Mnésiloque introduit, au lieu de la neige moelleuse fondante, la $\sigma \alpha \rho \mu \alpha i \alpha$, une

$\begin{array}{ll}\text { méta-thêâtre } & \text { comédie } \\ 855-857 & 846-854 \\ 859 * & 858 \\ 864 * & 860-863 \\ 866-867 * & 865 \\ 869-873 & 868 \\ 877-878 & 874-876 \\ 881 & 879-880 \\ 885-886 & 882-884 \\ 889-891 & 887-888 \\ 895-896 * & 892-894 \\ 899-915 * & 897-898 \\ 918-919 & 916-917 \\ & 920-928\end{array}$

On remarquera que la fiction méta-théâtrale est interrompue au moins cinq fois par les répliques insolentes de la femme (v. $860,865,897,916)$, tandis que la parodie ne l'emporte jamais sur le dialogue comique à la clausule. Cela revient à dire que la partie la plus importante et saillante du vers, la finale, est exclusivement réservée aux plaisanteries de la femme pour laquelle l'habileté poétique du duo Euripide / parent ressemble plutôt à une tromperie qu'à une manifestation artistique. En outre, la scène débute (v. 846-54) et s'achève (v. 920-8) sur le ton du dialogue à trois voix entre la femme de plus en plus agacée et les deux prisonniers désespérés et affligés par leur condition misérable et sans issue.

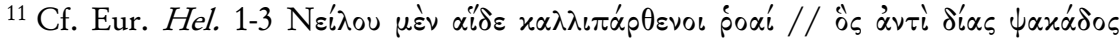

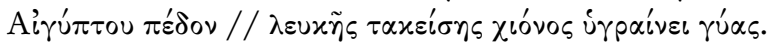


longue robe dont la couleur noire contraste avec le blanc de la couche de neige couvrant le sol égyptien ${ }^{12}$. Le parent ne touche absolument pas à la clausule dissyllabique de la version originale $\left(\gamma u^{\prime} \alpha \varsigma\right)$ et il se borne à la remplacer par $\lambda \varepsilon \varepsilon^{\prime} \omega \nu$ qui rime, au niveau de l'accent, avec le poo í du premier vers. Quant au schéma final, Mnésiloque s'en tient aussi au style tragique : les trois trimètres présentent les finales très communes mot de trois demi-pieds + dissyllabe, précédées par l'hephthémimère, et mot de cinq demi-pieds + dissyllabe, suivant la penthémimère. La femme, quant à elle, lui répond brutalement en le traitant de voyou :

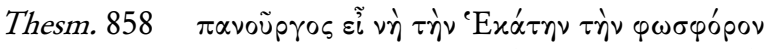

Tu es un fourbe, par Hécate la porte-lumière

Non seulement l'insulte détonne avec la solennité de la métaphore alambiquée du Nil, mais la clausule et le syntagme long de l'invocation à Hécate se placent aussi dans un schéma basé sur deux mots de quatre demi-pieds qui découpent la matière verbale du vers $x \alpha \tau \dot{\alpha} \delta \imath \pi \circ \delta^{\prime} \iota \alpha$. Le parent reprend la fiction en citant les v. 16-17 de l'Hélène, mais, soudainement, il est interrompu et injurié par la femme :

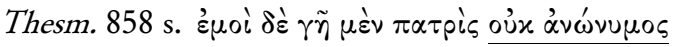

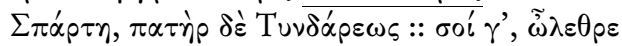

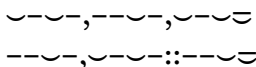

"J'ai pour patrie une célébre terre,

Sparte, et Tyndare est mon père » :: (femme) À toi, malheureux!

Le couplet réunit, côte à côte, les deux clausules emblématiques de la

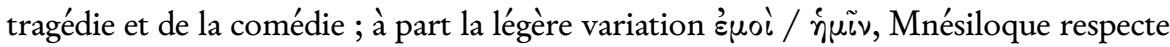
le modèle tragique et se drape dans un syntagme grandiloquent qui désigne la patrie de la protagoniste. Contrairement au texte de la tragédie d'Euripide qui évoque la légende des amours de Zeus et Léda, Aristophane ne doute pas que Tyndare soit le père d'Hélène. Au contraire, le nom de Tyndare donne l'occasion à la femme pour couper brusquement la parole de Mnésiloque et l'insulter par une clausule typiquement comique (trisyllabe et violation du pont de Porson). La femme poursuit ses injures en soulignant les origines humiles du parent (v. 861 avec une clausule monosyllabique) et, lorsqu'elle l'entend se présenter comme Hélène, elle n'arrive plus à contenir sa haine face à un voyou qui ose se présenter comme un parangon de vertu ( $\mathrm{p}=$ parent $; \mathrm{f}=$ femme) :

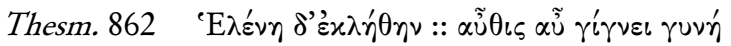

(p) «Je fus nommée Hélène » :: (f) Voilà encore que tu redeviens femme

${ }^{12}$ Cf. H. Frisk, 1970, p. 823. 
La déclaration (reprise littérale du v. 22 de l'hypotexte) de l'identité laconique faite par Mnésiloque est promptement tournée en dérision par la vanne lancée par la femme. La protagoniste effarée et plongée dans la douleur extrême de l'exilée n'est rien d'autre qu'un homme qui, tant bien que mal, porte des haillons rapiécés ; ce sont les mots poignants de la femme qui, à l'aide d'un schéma morcelé $(2+1+2+2)$, donne à la déclamation lyrique de Mnésiloque l'aspect d'une

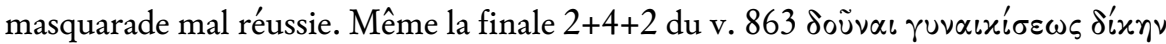
ne saurait être qu'une mauvaise copie du tragique $2+3+2$ avec hyperbate encadrante $\mathrm{ABA}^{13}$. Le constraste entre registre tragique et comique traverse la suite des répliques :

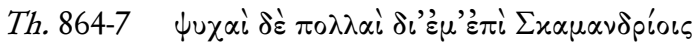

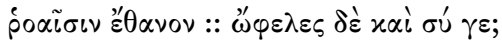

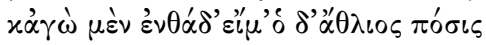

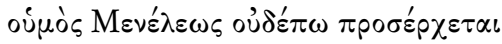
$\tau i$ oũv $\varepsilon \tau_{\imath} \zeta \tilde{\omega}$;

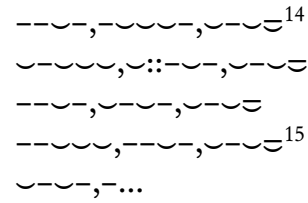

Dans l'évocation des dégâts matériels et humains dont elle se sent responsable, Mnésiloque / Hélène conclut la citation par un mot long inséré dans un syntagme solennel, en conférant à sa réflexion un caractère décidément lyrique. Mais, aux yeux (et aux oreilles) de la femme, Mnésiloque paraît bavarder à tort et à travers ; elle oppose alors une contre-attaque encore plus éhontée. La citation se poursuit ; bien que Mnésiloque extériorise ses angoisses face à la perte de son époux Ménélas, la femme ne maitrise plus sa colère, forcée à entendre de telles balivernes (v. 865). Au trimètre suivant, le parent revient sur l'état de refugiée d'Hélène, en contrefaisant le v. 49 de la tragédie euripidéenne ; à partir du v. 866 (la citation n'est plus littérale), l'explication de la guerre menée par Ménélas débouche sur l'immédiateté de la situation présente (clausule tragique $3+4$ adverbe + adverbe suivie de la reprise à la lettre du début du v. 56 du modèle). Le v. 868 constitue un

${ }^{13}$ Les chiffres symbolisent les demi-pieds de l'étendue du mot (e.g. 3+2 = mot de trois demi-pieds suivi d'un mot de deux demi-pieds) ; dans notre thèse sur La clausule dans le trimètres et sénaires iambiques grecs et latins (vol. 1, 362-365 ; à paraitre), on a démontré que la structure verbale $2+3+2$, où les deux mots de deux demi-pieds sont unis par un hyperbate, est typique du trimètre de la tragédie.

${ }^{14}$ Scander le v. 864 sans abrègement devant le groupe consonantique $\sigma x-$; cf. M.C. Martinelli, 1995, p. 54.

15 (Parent) "Bien de guerriers à cause de moi près des rives // du Scamandre 》 :: (femme) Qu'en es-tu mort, toi aussi ?" // (parent) "Je suis ici, mais lui, mon malheureux époux, // le pauvre Ménélas n’arrive toujours pas // pourquoi donc vis-je encore?". Scander le v. 867 sans synizèse. 
tournant décisif : en effet, la dernière plaisanterie de la femme (la punchline $\tau \tilde{\omega} \nu$

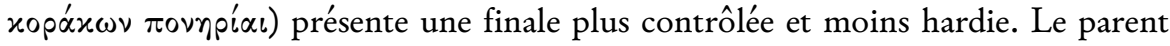
répond alors sur un rythme qui n'a rien de tragique :

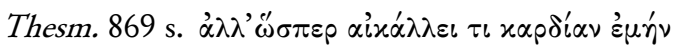

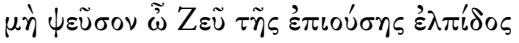
$--\smile-,--\smile /-,--\smile=$ "Mais quelque chose vient comme flatter mon cour.

$\hat{O}$ Zeus, ne démens pas l'espoir qui naît en moi »

Cette fois-ci, non seulement la citation de Mnésiloque est confuse et reprend la prière de Pélée chez Sophocle (fr. 493 R), mais aussi l'acteur se laisse emporter par la vivacité du dialogue comique et emploie une cheville sûrement peu tragique (4 avec résolution +3 avec violation du pont de Porson, atténuée par le syntagme soudé).

C'est alors qu'Euripide va entrer sur scène, vêtu en Ménélas naufragé :

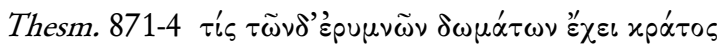

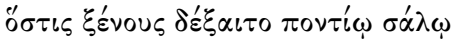

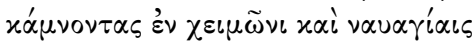
$--\smile-,--\smile-, \smile-\smile=$

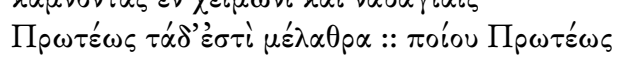
$--\smile-,--\cup-,--\smile=$ (Euripide) "Qui détient le pouvoir en ces fortes demeures?

Puisse-t-il accueillir des hôtes fatigués par la houle marine au cours d'une tempête et des pauvres naufragés!» (p) «Protée a ce palais » :: (f) Quel Protée ?!

Le premier vers est une reprise directe du v. 68 de l'Hélène où toutefois non pas Ménélas, mais Teucros demande des renseignements avant de rencontrer Hélène ; les deux vers suivants, quant à eux, constituent une transition, toujours en style tragique (schémas $3+2+2 / 3+2$ et 5 ), qui comprime l'action du modèle. En effet, en guise de réponse, Mnésiloque / Hélène fournit des explications que, dans la tragédie, la vieille bourrue donne à Ménélas. Mais Euripide saute le long dialogue entre Teucros et Hélène. Le v. 874 est partagé entre la réponse brève du parent (reprise ad sensum d'Hel. 460) et la question de stupeur et brutale de la femme (schéma $2+3$ avec violation du pont de Porson).

Dans la suite, toutes les interventions de la femme sont marquées par l'emploi de clausules typiquement comiques :

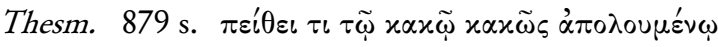

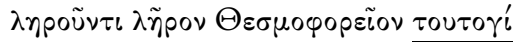

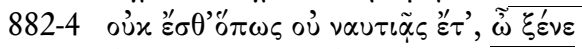

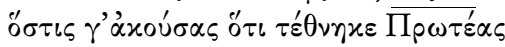

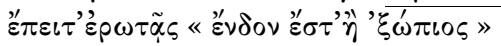

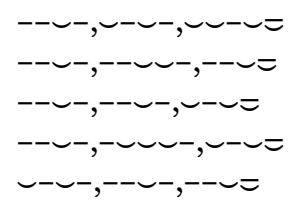




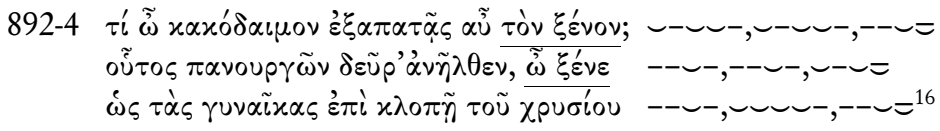

Comme on le voit, sur huit trimètres au moins cinq se terminent sur un trisyllabe dont deux (v. 880 et 892 ) engendrent une violation du pont de Porson. Le v. 879 réplique, certes, les trimètres de la tragédie à diérèse médiane, mais avec une uariatio importante : le tétrasyllabe final débute sur une résolution, une licence bannie par les Tragiques en cas de schéma $2+4$. Et, si le v. 884 répète les mots prononcés par Euripide/Ménélas au v. 881, citation ad sensum du v. 467 de l'Hélène, le schéma commun $3+4$ du dernier trimètre est associé à une autre résolution dans le

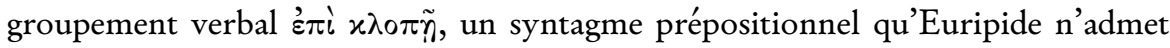
généralement qu'à la clausule. Bien que le duo des acteurs s'efforce toujours avec la même vigueur de reconstruire la fiction de l'Hélène, la femme réitère les piques à la clausule en traitant Mnésiloque / Hélène d'étranger. Entrecoupées par les insultes de la femme, les citations, désormais ad sensum et non plus à la lettre, enchaînent des schémas qui relèvent du style tragique : v. 885 s. $1+4+2$ et $2+4$ sans résolution ; v. 889-891 2+3+2 trois fois et 4 . Après la dispute avec les femmes, Euripide et le parent se lancent dans un passage de citations tragiques en stichomythie (v. $895 \mathrm{~s}$. et 899-919). Ici, une allure tragique imprègne toutes les clausules, ne serait-ce que pour le début du tac au tac qui précède la reconnaissance :

\begin{tabular}{|c|c|}
\hline \multirow{9}{*}{$\begin{array}{l}\text { Thesm. } \\
899-909\end{array}$} & \\
\hline & 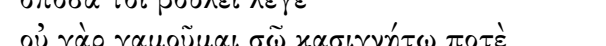 \\
\hline & 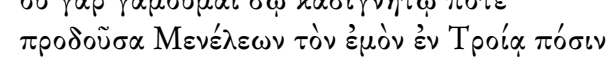 \\
\hline & 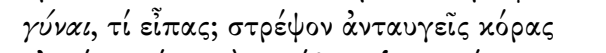 \\
\hline & 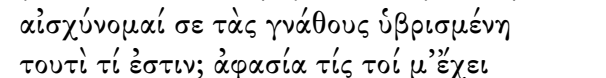 \\
\hline & 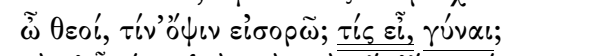 \\
\hline & 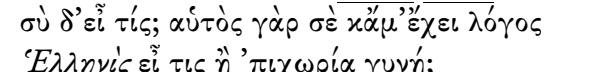 \\
\hline & 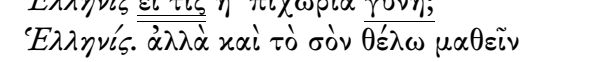 \\
\hline & 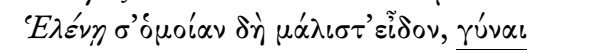 \\
\hline
\end{tabular}

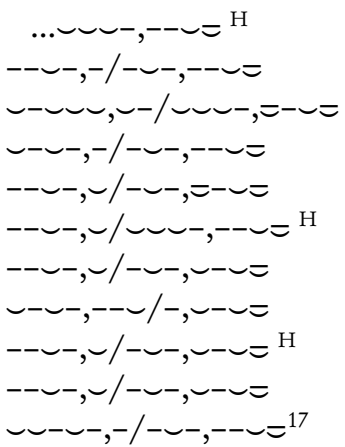

${ }^{16}$ Crois-tu cet homme digne de male mort // qui te conte des contes? C'est le Thesmophorion, ceci! // ...Il faut que tu aies encore des nausées, étranger; // on te dit que Protée est mort, // après tu demandes : "Est-il dedans ou dehors?" // ...Pourquoi, misérable, tromper encore cet étranger? // Cet homme est venu ici en fourbe, étranger, // afin de voler aux femmes leurs bijoux!

${ }_{17}$ (Parent) "Dis ce que tu voudras ; // je n'épouserai point ton frère // et n'irai pas trahir Ménélas, mon époux en Troade " // (Euripide) "Femme, qu'as-tu dit? Tourne les 
Dans un contexte de mémoire tragique indéniable, la dimension actuelle de la reconnaissance fait irruption au beau milieu de la fiction méta-théâtrale ; la stichomythie animée a pour but de souligner le crescendo incessant du pathos, où l'absence de parole s'accompagne de l'abasourdissement manifeste des deux amoureux. Les trimètres s'échelonnent les uns après les autres fondés sur la répétition ininterrompue des

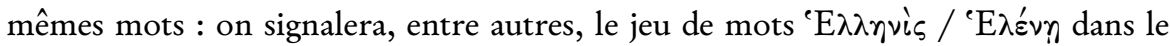
tac au tac qui mène à découvrir la vraie identité d'Hélène. À la clausule, le mot femme revient à plusieurs reprises justifier la présence du parent au Thesmophorion. Si, jusqu'au v. 906 la citation se fait ad sensum, à partir du vers suivant la citation devient littérale, ainsi $906=558,907-912=561-6$, en survolant le couplet en stichomythie où Ménélas soulignait la ressemblance de la femme à Hélène. L'élan pathétique qui précède la reconnaissance prend le dessus sur l'attachement au modèle et aux stylèmes tragiques. À Euripide / Ménélas qui dit avoir reconnu dans l'inconnu son épouse Hélène, Mnésiloque / Hélène répond avec un fulmen in clausula, qui remet en cause même la vieille plaisanterie sur la mère marâichère $d u$ Tragique $^{18}$. Au moment même de se retrouver, les deux acteurs / amants n'arrivent plus à contenir leur joie et la fiction méta-théâtrale qu'ils ont mise en œuvre :

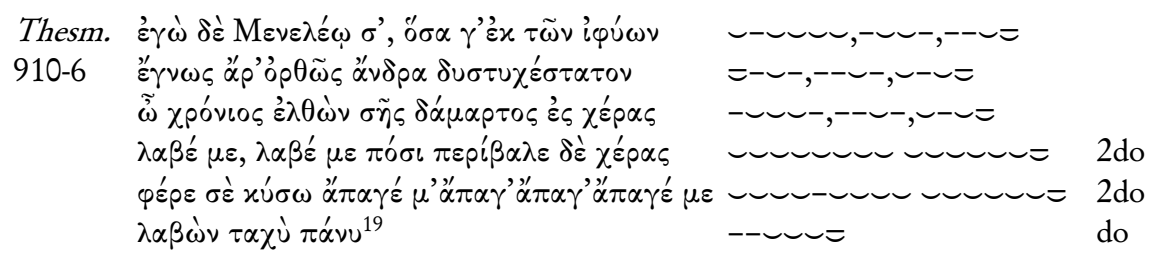

Dans le couplet du v. 910 s., le duo s'évertue à garder une structure du trimètre la plus possible proche du modèle tragique. Mais, juste après, la récitation par cœur dérape complètement en débouchant sur une scène ridicule, où les résolutions

yeux vers moi! " // (parent) "J'ai honte, après l'affront imprimé sur mes joues " // (Euripide) "Qu'est-ce là? La stupeur m'empêche de parler, // dieux, quel objet perçois-je? Toi, qui es-tu, femme?" // (parent) "Et toi, qui es-tu? Car je te dis même chose! " // (Euripide) "Grecque ou quelque femme habitant ce pays?" // (parent) "Grecque. Et toi ? Je veux le savoir à mon tour » // (Euripide) "Femme, tu es le portrait craché d'Hélène ».

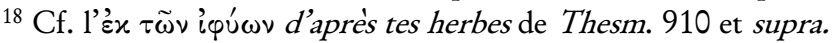

19 "Je te reconnais Ménélas, au moins d'après...tes herbes!" // " Cela est reconnaître un homme infortuné " // "Enfin tu es venu dans les bras de ta femme! // Oh, prendsmoi, prends-moi donc, embrasse-moi, embrasse-moi, chère âme, // allons, que je t'embrasse! Emmène, emmène-moi, emmène, emmène, emmène-moi, // emmène-moi bien vite!». 
contiguës des dochmies sont, pour reprendre les mots de B. Zimmermann, Ausdruck der Wiedersehensfreude ${ }^{20}$. C'est à nouveau le héros comique, Mnésiloque, l'homme à tout faire, le parent fidèle, qui transforme la fiction tragique en réalité comique. Celle-ci devient d'autant plus évidente dans la partie du dialogue final qui suit la parodie de l'Hélène, que les derniers trimètres reprennent, à un plus haut degré, la clausule trisyllabique :

Thesm.

925-8
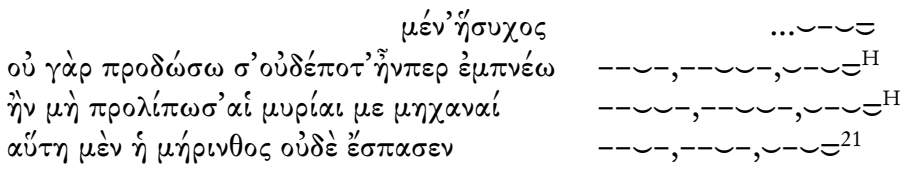

En plein dialogue comique, les clausules enchainent plusieurs trisyllabes finaux consécutifs ainsi que des schémas morcelés, propres à la comédie $(1+3,2+3$ deux fois ainsi que 4+3). Face à la promesse d'Euripide de revenir à l'action et de faire étalage de ses mille expédients artistiques (v. 927 ai $\mu \cup \rho i \alpha \iota \mu \varepsilon \mu \eta \chi \alpha \nu \alpha i)$, le parent se montre, au contraire, totalement dépité et se résigne à la punition du prytane qui est en train d'arriver.

La scène de la parodie de l'Hélène des Thesmophories montre donc à quel point la clausule constitue un atout stylistique pour soutenir le passage du registre solennel de la tragédie au registre familier de la comédie. La femme, tout en s'arcboutant sur ses préjugés, réussit dans son objectif de reporter à la réalité une fiction aussi para-tragique que ridicule.

\section{CONCLUSION}

Trois scènes para-tragiques tirées des Guêpes (le concours de chant), des Grenouilles (la pesée des vers) et des Thesmophories (parodie de l'Hélène) d'Aristophane ont montré le réseau de correspondances sémantiques que la clausule tisse avec les personnages et leur parole. L'alternance de clausules plus tragiques à d'autres plus comiques répond bien au contenu para-tragique, à la volonté de détournement de la tragikè lexis des passages en question. Et c'est précisément là que cette étude apporte un élément fondamental, nouveau : l'existence pour la tragédie et la comédie de clausules différentes que les passages les plus para-tragiques d'Aristophane, où le dialogue entre les deux registres est tellement continu, font rejaillir.

${ }^{20}$ Cf. B. Zimmermann, 1985, p. 61.

21 (Euripide) ...reste tranquille, // car jamais je ne te trahirai, tant que j'aurai un souffle de vie // à moins que mes innombrables ruses ne viennent à me manquer! // (parent) Cette ligne à pêcher-là n'a rien tiré! 
6. BIBLIOGRAPHIE

G. Barabino, 1978, "Nota sul verso asclepiadeo » dans Problemi di metrica classica. Miscellanea filologica, Università di Genova, Facoltà di Lettere (éd.), Genova, pp. 163-177.

H. Frisk, 1970, Griechisches etymologisches Wörterbuch. Band II: Kp -, , Heidelberg.

J. Irigoin, 1956, « La structure des vers éoliens », $A C$ 25, pp. 5-19.

M.C. Martinelli, 1995, Gli strumenti del poeta: elementi di metrica greca, Bologna.

M. Steinrück, 2007, À quoi sert la métrique? Interprétation littéraire et analyse des formes métriques grecques : une introduction, Grenoble.

J. Veremans, 1976, «L'asclépiade mineur chez Horace, Sénèque, Terentianus Maurus, Prudence, Martianus Capella et Luxorius », Latomus 35, 12-42.

B. Zimmermann, 1985, Untersuchungen zur Form und dramatischen Technik der Aristophanischen Komödien. Band 2. Die anderen lyrischen Partien, Königstein/Ts.

B. Zimmermann, 1987, Untersuchungen zur Form und dramatischen Technik der Aristophanischen Komödien. Band 3. Die metrischen Analyse, Frankfurt am Main. 